Research Article

\title{
Predictors of Treatment Outcomes among Pediatric Patients Hospitalized with Pneumonia in Tikur Anbessa Specialized Hospital, Addis Ababa, Ethiopia
}

\author{
Hilina Tsegaw, ${ }^{1}$ Mohammed Yimam, ${ }^{2}$ Dejen Nureye $\mathbb{D}^{1},{ }^{1}$ Workineh Woldeselassie, ${ }^{1}$ \\ and Solomon Hambisa $\mathbb{1}^{3}$ \\ ${ }^{1}$ School of Pharmacy, College of Medicine and Health Sciences, Mizan-Tepi University, Mizan-Teferi, Ethiopia \\ ${ }^{2}$ Department of Pharmacy, College of Health Sciences, Debre Brehan University, Debre Brehan, Ethiopia \\ ${ }^{3}$ Department of Pharmacy, College of Medicine and Health Sciences, Ambo University, Ambo, Ethiopia \\ Correspondence should be addressed to Solomon Hambisa; s.hambisa@gmail.com
}

Received 10 December 2020; Revised 12 March 2021; Accepted 10 April 2021; Published 20 April 2021

Academic Editor: Abdelhakim Bouyahya

Copyright ( $) 2021$ Hilina Tsegaw et al. This is an open access article distributed under the Creative Commons Attribution License, which permits unrestricted use, distribution, and reproduction in any medium, provided the original work is properly cited.

Background. Pneumonia remains the leading cause of hospitalization and mortality in young children in low- and middle-income countries. This study is aimed to assess predictors of treatment outcomes among pediatric patients hospitalized with pneumonia in Tikur Anbessa Specialized Hospital, Addis Ababa, Ethiopia. Methods. A facility-based retrospective cross-sectional study was conducted among pediatric patients admitted with pneumonia, considering patient medical charts recorded for a 1-year period from February 2017 to February 2018. The sample size was computed based on a single population proportion formula and giving a total sample size of 207. The systematic random sampling method was employed to select patient cards from the sampling frame. The data extraction format was used to extract any relevant information from patient chart. The processed data were analyzed by using Statistical Package for Social Sciences (SPSS, version 21). Descriptive statistics were used to summarize the patients' baseline characteristics and related information. The logistic regression model was fitted to determine factors associated with treatment outcomes. To identify predictors of poor treatment outcome, the level of significance was set at $P<0.05$. Results. From a total of 207 patient charts reviewed, more than half (55.6\%) of the study participants were males. Majority of patients, 130 (62.8\%), were in the age range of 1 month-11 months. Furthermore, 191 (92.3\%) patients had good treatment outcome. Patients who treated with ceftriaxone + azithromycin were less likely to have poor treatment outcome compared with patients who were placed on crystalline penicillin $(\mathrm{AOR}=0.86,95 \% \mathrm{CI} 0.01-0.83$ ). On the contrary, patients who stayed $\geq 8$ days were about 14.3 times more likely to have poor treatment outcome compared with patients who stayed $\leq 3$ days (AOR $=14.3,95 \%$ CI 1.35-151.1). Conclusion. Even though the study revealed good treatment outcome among the pediatric patients, particular consideration should be given to children in need of other interventions.

\section{Background}

Pneumonia is a severe form of acute respiratory infection known to affect the lungs $[1,2]$. It remains the leading cause of hospitalization and mortality in young children in lowand middle-income countries as per the 2016 UNICEF annual report and is estimated to cause 896,000 deaths of the total 5.6 million deaths $[3,4]$. In Ethiopia, pneumonia is a substantial cause of morbidity and mortality in the pediatric population, and regardless of improved preventive strategies, approximately 4 million childhood pneumonia cases are occurring annually [5].

A number of infectious agents are responsible for childhood pneumonia, including viruses, bacteria, and fungi $[1,2]$. The respiratory syncytial virus, Streptococcus pneumoniae, and Haemophilus influenzae are the leading causes of childhood pneumonia, with the latter two being preventable through administration of vaccines [6]. The main risk factors for pediatric pneumonia include poor socioeconomic status, not being able to breastfed, malnutrition, 
indoor air pollution, household crowding, low birth weight, incomplete immunization schemes, HIV, prolonged duration of illness, and the presence of underlying chronic illness such as underlying heart disease [7-9].

Although under-five mortality was consistently reduced over the past 20 years, few progresses in reducing neonatal mortality have been done [10]. Pediatric patients who receive effective and timely treatment recover without complication. However, patients with no access to effective intervention are at high risk of developing complications and death. The major complications of pneumonia include lung abscess, pleural effusion and empyema, and pneumothorax [11].

Most guidelines recommend the use of a combination of $\beta$-lactam antibiotics with a macrolide or monotherapy with respiratory fluoroquinolone, but not $\beta$-lactam monotherapy in treatment of pneumonia cases. This is especially for patients who are admitted to medical ward of the health institutions [12]. Likewise, treatment regimens are needed to be chosen based on their efficacy in local settings; for instance, some areas may have a high burden of resistance to certain antibiotics, rendering these antibiotics are less effective for management of pneumonia. It is also recommendable to consider high-risk groups, such as malnourished or HIV-positive children to adapt their treatment approaches according to their medical cases for the better outcome $[13,14]$.

Despite the availability of effective management and treatment tools for pneumonia [15], poor response to initial empirical treatment of community-acquired pneumonia represents a major challenge for clinicians and requires early identification and intervention. The incidence of treatment failure in community-acquired pneumonia (CAP) is 10 to $15 \%$, and the mortality is increased nearly fivefold. Resistant and unusual microorganisms and noninfectious causes are responsible for the treatment failure. Risk factors for the poor treatment outcomes are related to the initial severity of the disease, the presence of comorbidity, the microorganism involved, and the antimicrobial treatment administered during pharmacotherapy [16].

Several studies have reported variation in extent of treatment outcome among pediatric populations admitted with pneumonia $[5,17,18]$. Furthermore, the investigation of treatment outcome among patients with pneumonia would have paramount importance to demonstrate the extent of pneumonia management and care in health care settings [5]. Identifying factors associated with the treatment outcomes among pediatric patients hospitalized with pneumonia also play a crucial role for proper planning and intervention, particularly in developing countries like Ethiopia where prevalence of infectious diseases is high. Consequently, this study is aimed to assess predictors of treatment outcomes among pediatric patients hospitalized with pneumonia in Tikur Anbessa Specialized Hospital (TASH), Addis Ababa, Ethiopia.

\section{Methods and Materials}

2.1. Study Setting and Study Design. A facility-based retrospective cross-sectional study was conducted among pediatric patients hospitalized with pneumonia at TASH, Addis Ababa, Ethiopia. TASH is found in the capital city of Ethiopia, Addis Ababa, and it is among the oldest and pioneering teaching referral hospitals with a capacity of 700 beds. TASH has a range of specialties including pediatrics, surgery, gynecology, and psychiatry. The pediatric unit provides emergency service for patients who are referred from different health institutions, and it is the main portal of entry for children who need prompt admission and management.

2.2. Sample Size and Sampling Procedure. For this particular study, patient medical charts recorded for a 1-year period from February 2017 to February 2018 were reviewed, and the sample size was computed based on the single population proportion formula, taking the assumption of 5\% margin of error, $95 \%$ confidence interval, and $16 \%$ prevalence of death from pediatric pneumonia [3] and giving a total sample size of 207. The systematic random sampling method was employed to select patient cards, and the sampling interval was calculated by dividing patient charts kept for one year by actual sample size.

Patients with age $\leq 14$ years admitted with clinical diagnosis of pneumonia in a pediatric ward of TASH were included in our study. Pediatric patients with uncertainty in the diagnosis of pneumonia, patients with incomplete medical records, patients who died before they start treatment, and patients referred to other health care facilities were excluded from the study.

2.3. Data Collection Tool and Procedure. The data extraction format was developed to extract any relevant information from patient chart regarding patient sociodemographic characteristics, clinical characteristics of the patients including diagnostic method(s) used for identification of the disease, pertinent laboratory findings, comorbidities, medications administered, type and severity of the disease, patient's discharge information (treatment outcome), and duration of hospital stay. Data collection was performed by well-trained three pharmacy professionals, and completeness of collected data was checked by the supervisors.

2.3.1. Data Analysis. The processed data were analyzed by using Statistical Package for Social Sciences (SPSS, version 21). Descriptive statistics such as frequency, percentage, and mean were used to summarize the patients' baseline characteristics and related information. The logistic regression model was fitted to determine factors associated with treatment outcomes. Variables with $P$ value $\leq 0.25$ on bivariate logistic regression were entered into multivariate logistic regression, and to identify predictors of poor treatment outcome, the level of significance was set at $P<0.05$.

2.3.2. Ethical Consideration. Ethical clearance was obtained from the Ethical review board of Mizan-Tepi University, College of Medicine and Health Sciences. Official permission 
to access patients' data was granted from the hospital management. Participants' information was kept confidential and anonymous. Patient medical charts were handled in an appropriate manner.

\subsection{Operational Definitions}

2.4.1. Treatment Outcome. Result was obtained after the use of a drug. It may be cure, death, and complication.

2.4.2. Good Treatment Outcome. It represents the discharge from hospital on the grounds of clinical improvement.

2.4.3. Poor Treatment Outcome. It is defined as the occurrence of death and complications or an intensive care unit (ICU) admission.

\section{Results}

3.1. Demographic Characteristics. From a total of 207 patient charts reviewed, more than half (55.6\%) of the patients were males. The majority of patients, $130(62.8 \%)$, were in the age range of 1 month to 11 months. Two-thirds (66.7\%) of the participants were weighed in the range of 6 to $10 \mathrm{~kg}$ (Table 1).

3.2. Clinical Characteristics of the Study Participants. Regarding the types of pneumonia, most patients (88.9\%) had been diagnosed with community-acquired pneumonia (CAP), followed by hospital-acquired pneumonia (HAP) (5.3\%). The severity of pneumonia was determined as severe pneumonia in most of the participants (88.9\%), while 21 (10\%) had been depicted as nonsevere one. From all pediatrics admitted with pneumonia, one hundred forty-four (69.6\%) were diagnosed merely based on complete blood count. However, around one-fourth (24.6\%) of the cases were confirmed together with chest radiography. Causative microorganisms were not identified in most patients (Table 2).

As presented in Table 2, the study revealed that the majority $(56.5 \%)$ of patients had comorbid diseases besides pneumonia. Congestive heart failure (CHF) and hyperactive airway disease (HAAD) were the most common comorbidities in the study population, which accounts for 41 (19.8\%) and 20 (9.7\%), respectively. Eighty-one (39\%) patients stayed in hospital for 4-5 days, while merely around one-fifth (17.4\%) of study participants were admitted in hospital beyond one week. The mean duration of the hospital stay was 2.52 days.

3.3. Medication-Related Characteristics. Patients were treated with a variety of parenteral antibiotics during their hospital stay. The most frequently used antibiotics were parenteral crystalline penicillin (111 (53.6\%)), followed by ceftriaxone (46 (22.2\%)). Paracetamol was the most commonly prescribed comedication $(27(11 \%))$. From the total number of children treated for pneumonia, most $(85 \%)$ of
TABle 1: Demographic characteristics of the children admitted with pneumonia at pediatric ward of Tikur Anbessa Specialized Hospital from February 2017 to February 2018, Addis Ababa, Ethiopia $(N=207)$.

\begin{tabular}{lc}
\hline Demographic variable & Frequency $(\%)$ \\
\hline Sex & \\
Male & $115(55.6 \%)$ \\
Female & $92(44.4 \%)$ \\
\hline Age & \\
$<1$ month & $2(1 \%)$ \\
1 month-11 months & $130(62.8 \%)$ \\
1 year- $<3$ years & $46(22.2 \%)$ \\
3 years-<6 years & $16(7.7 \%)$ \\
$>6$ years & $13(6.3 \%)$ \\
\hline Weight $(\mathrm{kg})$ & \\
$1-5 \mathrm{~kg}$ & $23(11.1 \%)$ \\
$6-10 \mathrm{~kg}$ & $138(66.7 \%)$ \\
$11-15 \mathrm{~kg}$ & $30(14.5 \%)$ \\
$>16 \mathrm{~kg}$ & $16(7.7 \%)$ \\
\hline
\end{tabular}

the participants were discharged with oral medications. Out of the total prescribed medications, amoxicillin-clavulanate (Augmentin) (57 (27.5\%)) and amoxicillin (56 (27.1\%)) were the most commonly prescribed oral medications (Table 3 ).

3.4. Factors Associated with Treatment Outcome. Out of 207 pediatric patients, the majority $(191(92.3 \%))$ of them were found to have a good treatment outcome. In contrary, 16 (7.7\%) of the participants had a poor treatment outcome (Table 2). The results of univariate logistic regression analysis depicted that patients age group between 1 year and 3 years $(\mathrm{COR}=0.022,95 \% \mathrm{CI}: 0.001-0.67)$, patients whose weight was between 6 and $10 \mathrm{~kg}(\mathrm{COR}=0.22,95 \% \mathrm{CI}$ : $0.05-0.67)$, patients who were treated with vancomycin $(\mathrm{COR}=20.1,95 \%$ CI: 3.32-121.3), patients who were treated with ceftriaxone + azithromycin $\quad(\mathrm{COR}=7.6, \quad 95 \% \quad \mathrm{CI}$ : 1.8-49.2), patients who were treated with ceftriaxone + metronidazole $(\mathrm{COR}=10.7,95 \% \mathrm{CI}: 1.6-72.9)$, and patients who stayed in hospital beyond one week $(\mathrm{COR}=3.57,95 \%$ CI: $1.9-18.4)$ were significantly associated with poor treatment outcome.

Among the variables entered to multivariable logistic regression, treating patients with the ceftriaxone plus azithromycin and duration of hospital stay for $\geq 8$ days were independent predictors of poor treatment outcome. Accordingly, patients who were treated with ceftriaxone + azithromycin were less likely to have a poor treatment outcome compared with patients who were placed on crystalline penicillin (AOR $=0.86,95 \%$ CI: $0.01-0.83$ ). On the contrary, patients who stayed for $\geq 8$ days in hospital were 14.3 times more likely to have a poor treatment outcome compared with patients who stayed $\leq 3$ days in hospital $(\mathrm{AOR}=14.3,95 \%$ CI: 1.35-151.1) (Table 4).

\section{Discussion}

This study revealed a high proportion of good treatment outcome among pediatric patients diagnosed with 
TABLE 2: Clinical characteristics of the study participants admitted with pneumonia in pediatric ward of Tikur Anbessa Specialized Hospital from February 2017 to February 2018, Addis Ababa, Ethiopia.

\begin{tabular}{lc}
\hline Clinical variable & Frequency (\%) \\
\hline Comorbid disease & \\
No & $90(43.5 \%)$ \\
Yes & $117(56.5 \%)$ \\
\hline Specific comorbidities & \\
HAAD & $41(19.8 \%)$ \\
Anemia & $11(5.3 \%)$ \\
Malnutrition & $14(6.8 \%)$ \\
CHF & $20(9.7 \%)$ \\
AGE & $18(8.7 \%)$ \\
Others* & $13(6.3 \%)$ \\
\hline Severity class of pneumonia & \\
Very severe & $2(1 \%)$ \\
Severe & $184(88.9 \%)$ \\
Nonsevere & $21(10 \%)$ \\
\hline Types of pneumonia & \\
CAP & $184(88.9 \%)$ \\
HAP & $11(5.3 \%)$ \\
Aspiration pneumonia & $6(2.9 \%)$ \\
Recurrent pneumonia & $6(2.9 \%)$ \\
\hline Diagnostic method & \\
Complete blood count & $144(69.6 \%)$ \\
Complete blood count and chest radiography & $51(24.6 \%)$ \\
Culture & $10(4.8 \%)$ \\
Chest radiography & $2(1 \%)$ \\
\hline Hospital duration (days) & \\
$\leq 3$ & $27(13 \%)$ \\
$4-5$ & $81(39 \%)$ \\
6-7 & $63(30.4 \%)$ \\
$\geq 8$ & $36(17.4 \%)$ \\
\hline Treatment outcome & \\
Discharged with improvement & $191(92.3 \%)$ \\
Occurrence of death and complications & $(5.3 \%)$ \\
Intensive care unit admission & $5(2.4 \%)$ \\
\hline & \\
\hline & \\
\hline &
\end{tabular}

${ }^{*}$ HIV/AIDS, hypertension, pyogenic meningitis, and disseminated TB.

pneumonia. This finding is comparable to the multicenter study done in the United States of America, in which 97.6\% of patients showed clinically significant improvements [17]. However, the finding is higher than the result that came out from Rabat, Morocco, where $72.8 \%$ of children had a poor prognosis [18]. Similarly, the finding of the present study is higher than the result from the Nekemte Referral Hospital, Ethiopia, in which poor treatment was observed in $69.4 \%$ of pediatric patients [5]. This discrepancy might have been related to the variation in the level of care given by the hospitals for the patients.

On the contrary, poor treatment outcome reported in some pediatric patients could be due to the fact that most patients visiting TASH are referred from other neighboring hospitals and terminally ill. Likewise, the presence of a high proportion of comorbidities like HIV, TB, heart failure, and anemia may result to poor prognosis. It is also interesting to note that both good treatment outcome and poor treatment outcome revealed in the study were within the minimum
TABLE 3: Patterns of medication utilization among children treated for pneumonia at the pediatric ward of Tikur Anbessa Specialized Hospital from February 2017 to February 2018, Addis Ababa, Ethiopia.

\begin{tabular}{lc}
\hline Patterns of medication utilization & Frequency $(\%)$ \\
\hline Types of antibiotic(s) administered & \\
Crystalline penicillin & $11(53.6 \%)$ \\
Ampicillin + gentamycin & $12(5.8 \%)$ \\
Ceftriaxone & $46(22.2 \%)$ \\
Ceftriaxone + gentamycin & $15(7.2 \%)$ \\
Ceftriaxone + azithromycin & $9(4.3 \%)$ \\
Vancomycin & $7(3.4 \%)$ \\
Ceftriaxone + metronidazole & $7(3.4 \%)$ \\
\hline Comedication & \\
No & $71(34.3 \%)$ \\
Yes & $136(65.7 \%)$ \\
\hline Types of comedication & \\
Paracetamol & $53(25.6 \%)$ \\
Salbutamol puff & $19(9.2 \%)$ \\
Hydrocortisone and salbutamol & $24(11.6 \%)$ \\
Furosemide and spironolactone & $10(4.8 \%)$ \\
Others* & $30(14.8 \%)$ \\
\hline Patient discharge medication & \\
No & $31(15 \%)$ \\
Yes & $176(85 \%)$ \\
\hline Types of discharge medication & \\
Amoxicillin-clavulanate & $57(27.5 \%)$ \\
Amoxicillin syrup & $56(27.1 \%)$ \\
Amoxicillin + paracetamol & $14(6.8 \%)$ \\
Amoxicillin + salbutamol puff & $14(6.8 \%)$ \\
Amoxicillin-clavulanate + salbutamol puff & $7(3.4 \%)$ \\
Amoxicillin-clavulanate + paracetamol & $10(4.8 \%)$ \\
Others & $18(8.7 \%)$ \\
${ }^{*}$ Haem up syrup, rifampin, isoniazid, pyrazinamide, and ethambutol.
\end{tabular}

standard set of the WHO guideline for management of severe pneumonia [19].

For a large number of pediatric patients diagnosed with pneumonia, crystalline penicillin was prescribed followed by ceftriaxone injections. In connection to this, the result is higher than another study obtained from Ethiopia, in which crystalline penicillin was ordered for $36 \%$ of patients [5]. The variation might be attributed to the differences in the study settings. In TASH, patients are most of the time given a care by the specialists and those health care providers were trained at an advanced level. Furthermore, the higher utilization of crystalline penicillin and ceftriaxone attributed to the fact that these drugs have been recognized as a drug of choice owing to its excellent bioavailability, effectiveness, and low toxicity profile $[18,20,21]$. Moreover, a recent randomized controlled trial and a preceding systematic review revealed that $\beta$-lactam antibiotics alone may be as effective as respiratory fluoroquinolones or combination regimens in the treatment of hospitalized patients diagnosed with CAP [22, 23]. Conversely, this study demonstrated that only a small fraction of patients was treated with ceftriaxone plus azithromycin, which is an alternative treatment for the pneumonia caused by resistant pathogen as stipulated in the Ethiopian national treatment guideline [20]. 
TABle 4: Predictors of treatment outcomes among children treated for pneumonia at the pediatric ward of Tikur Anbessa Specialized Hospital from February 2017 to February 2018, Addis Ababa, Ethiopia.

\begin{tabular}{|c|c|c|c|c|c|}
\hline \multirow[t]{2}{*}{ Variable } & & \multicolumn{2}{|c|}{$\begin{array}{l}\text { Treatment } \\
\text { outcome }\end{array}$} & \multirow[t]{2}{*}{ Corollary (95\% CI) } & \multirow[t]{2}{*}{$\operatorname{AOR}(95 \% \mathrm{CI})$} \\
\hline & & Good & Poor & & \\
\hline \multirow{5}{*}{ Age } & $<1$ month & 1 & 1 & 1 & 1 \\
\hline & 1 month-1 year & 121 & 9 & $0.074(0.004-1.3)$ & $4.4(0.015-13.2)$ \\
\hline & 1 year-3 years & 45 & 1 & $0.022(0.001-.67)^{*}$ & $0.77(0.01-73.2)$ \\
\hline & 3 years -6 years & 14 & 2 & $0.143(0.006-3.3)$ & $0.27(0.003-26.8)$ \\
\hline & $>6$ years & 10 & 2 & $0.300(0.014-6.4)$ & $1.2(0.05-27.7)$ \\
\hline \multirow{4}{*}{ Weight (kg) } & $1-5 \mathrm{~kg}$ & 19 & 4 & 1 & 1 \\
\hline & $6-10 \mathrm{~kg}$ & 132 & 6 & $0.216(0.056-0.84)^{*}$ & $1.139(0.11-118.3)$ \\
\hline & $11-15 \mathrm{~kg}$ & 28 & 2 & $0.339(0.056-2.041)$ & $0.2 .43(0.03-17.6)$ \\
\hline & $>16 \mathrm{~kg}$ & 12 & 4 & $1.58(0.332-7.56)$ & $0.396(0.013-12.4)$ \\
\hline \multirow{7}{*}{ Antibiotic administered } & Crystalline penicillin & 107 & 4 & 1 & 1 \\
\hline & Ampicillin + gentamycin & 11 & 1 & $2.43(0.25-23.7)$ & $1.83(0.072-46.57)$ \\
\hline & Ceftriaxone & 43 & 3 & $1.86(0.40-8.6)$ & $1.61(0.24-10.96)$ \\
\hline & Ceftriaxone + gentamycin & 14 & 1 & $1.91(0.19-18.3)$ & $0.887(0.08-9.4)$ \\
\hline & Ceftriaxone + azithromycin & 7 & 2 & $7.6(1.8-49.2)^{*}$ & $0.086(0.01-0.83)^{*}$ \\
\hline & Vancomycin & 4 & 3 & $20.1(3.32-121.3)^{*}$ & $0.123(0.011-1.35)$ \\
\hline & Ceftriaxone + metronidazole & 5 & 2 & $10.7(1.6-72.9)^{*}$ & $0.241(0.024-2.4)$ \\
\hline \multirow{4}{*}{ Hospital stay (days) } & $\leq 3$ & 25 & 2 & 1 & 1 \\
\hline & $4-5$ & 77 & 4 & $0.65(0.112-3.76)$ & $0.828(0.137-5.01$ \\
\hline & $6-7$ & 61 & 2 & $0.41(0.055-31)^{*}$ & $3.73(0.59-23.4$ \\
\hline & $\geq 8$ & 28 & 8 & $3.57(1.9-18.4)$ & $14.30(1.35-51.1)^{*}$ \\
\hline
\end{tabular}

In the present study, treating pediatric patients with ceftriaxone and azithromycin was significantly associated with poor treatment outcome in the univariate and multivariate logistic regression models. It was observed that patients who treated with ceftriaxone + azithromycin were less likely to have a poor treatment outcome compared with patients who were placed on crystalline penicillin. Several studies have revealed that, in severely ill communityacquired pneumonia patients, adding a macrolide to the antibiotic regimen is associated with better clinical outcomes $[24,25]$. Furthermore, macrolide antibiotics should be used if either mycoplasma or chlamydia pneumonia is suspected or in very severe disease [26]. Similarly, ceftriaxone is recommended for beta-lactamase-producing Haemophilus influenzae and rapidly progressing multilobar disease or pneumatoceles [27].

It was highlighted that a mean duration of hospitalization in our study was shorter. This study finding is lower as compared to similar finding that came out from New Delhi, India, where the mean length of hospital stay was 6.8 days [28]. Prolonged hospitalization was a significant predictor of poor treatment outcome. It was observed that patients hospitalized for $\geq 8$ days were about 14.3 times more likely to have a poor treatment outcome compared with patients admitted for $\leq 3$ days. This could be due to the fact that longer duration of hospital stay could be related to overcrowding, higher risk of developing hospital-acquired infection, and complications of bacterial pneumonia like persistent effusions, empyema, pulmonary abscess, and respiratory distress sepsis. Besides, the finding is slightly concurrent with the study done in Rabat, Morocco, that reported 9.96 days of hospital stay were significantly $(P=0.001)$ associated with poor treatment outcomes [18].

\section{Limitation of the Study}

The study has several limitations that should be taken into account while interpreting the results. As the data were collected retrospectively, a result does not show a strong causal effect between the variables of interest. The study was conducted at only a single facility, so caution should be taken in extrapolating the results of the study. A larger-scale and multicenter survey that includes more diverse participants is needed to provide more accurate finding.

\section{Conclusion}

The present study revealed that the majority of the pediatric patients admitted with pneumonia had a good treatment outcome. Treatment with ceftriaxone and azithromycin and prolonged hospitalization were a significant predictor of treatment outcome. Patients treated with ceftriaxone plus azithromycin showed a better treatment outcome than patients treated with crystalline penicillin alone. Longer duration of hospital stay was more likely associated with poor prognosis. Therefore, particular consideration should be given to children in need of other interventions.

\section{Abbreviations}

AOR: Adjusted odds ratio

AGE: Acute gastroenteritis

CAP: Community-acquired pneumonia

CI: $\quad$ Confidence interval

COR: Crude odds ratio

CHF: Congestive heart failure

HAP: Hospital-acquired pneumonia 
HAAD: Hyperactive airway disease

ICU: Intensive care unit

TASH: Tikur Anbessa Specialized Hospital

TB: Tuberculosis.

\section{Data Availability}

The authors declare that the supporting data on which conclusions of this manuscript are fully described within the manuscript.

\section{Conflicts of Interest}

The authors declare that they have no conflicts of interest.

\section{Acknowledgments}

The authors are grateful to Mizan-Tepi University, College of Medicine and Health Sciences, for providing an opportunity to conduct the research. The authors are also heartily thankful to TASH staff members and data collectors for their contribution.

\section{References}

[1] D. Roth, L. E. Caulfield, M. Ezzati, and R. E. Black, “Acute lower respiratory infections in childhood: opportunites for reducing the global burden through nutritional interventions," Bulletin of the World Health Organization, vol. 86, no. 5, pp. 356-364, 2008.

[2] J. Bryce, C. Boschi-Pinto, K. Shibuya, and R. E. Black, "WHO estimates of the causes of death in children," The Lancet, vol. 365, no. 9465, pp. 1147-1152, 2005.

[3] UNICEF, Levels \& Trends in Child Mortality, UNICEF, New York, NY, USA, 2017.

[4] C. L. F. Walker, I. Rudan, L. Liu et al., "Global burden of childhood pneumonia and diarrhoea," The Lancet, vol. 381, no. 9875, pp. 1405-1416, 2013.

[5] G. Bayisa, Y. Likey, and B. Mosisa, "Clinical treatment outcomes of pneumonia among hospitalized pediatric patients in Nekemte referral hospital, pediatrics ward, Ethiopia," World Journal of Pharmacy and Pharmaceutical Sciences, vol. 6, no. 2, pp. 68-84, 2017.

[6] D. T. Leung, M. J. Chisti, and A. T. Pavia, "Prevention and control of childhood pneumonia and diarrhea," Pediatric Clinics of North America, vol. 63, no. 1, pp. 67-79, 2016.

[7] F. Shann, J. Barker, and P. Poore, "Clinical signs that predict death in children with severe pneumonia," The Pediatric Infectious Disease Journal, vol. 8, no. 12, pp. 852-855, 1989.

[8] I. Rudan, C. Boschi-Pinto, Z. Biloglav, K. Mulholland, and H. Campbell, "Epidemiology and etiology of childhood pneumonia," Bulletin of the World Health Organization, vol. 86, no. 5, pp. 408-416, 2008.

[9] S. Jackson, K. H. Mathews, D. Pulanic et al., "Risk factors for severe acute lower respiratory infections in children: a systematic review and meta-analysis," Croat Med J, vol. 54, no. 10, pp. 110-121, 2013.

[10] UNICEF, Pneumonia the Forgotten Killer of Children, UNICEF, New York, NY, USA, 2014.
[11] G. Mitike, M. Addissie, M. Admassu et al., "Pneumonia in under-five children," East African Medical Journal, vol. 32, pp. 18-24, 2001.

[12] M. Woodhead, F. Blasi, S. Ewig et al., "Guidelines for the management of adult lower respiratory tract infectionsSummary," Clinical Microbiology and Infection, vol. 17, pp. 1-24, 2011.

[13] WHO and UNICEF, Joint Statement on Management of Pneumonia in the Community, WHO and UNICEF, New York, NY, USA, 2004.

[14] WHO, Technical Basis for the WHO Recommendations on the Management of Pneumonia in Children at First-Level Health Facilities, WHO, Geneva, Switzerland, 1991.

[15] G. Jones, R. W. Steketee, R. E. Black et al., "How many child deaths can we prevent this Year?" The Lancet, vol. 362, no. 9377, pp. 65-71, 2003.

[16] L. M. Gessman and D. I. Rappaport, Approach to CommunityAcquired Pneumonia in Children, WHO, Geneva, Switzerland, 2009.

[17] T. Q. Tan, E. O. Mason Jr., W. J. Barson et al., "Clinical characteristics and outcome of children with pneumonia attributable to penicillin-susceptible and penicillinnonsusceptible Streptococcus pneumoniae," Pediatrics, vol. 102, no. 6, pp. 1369-1375, 1998.

[18] I. Jroundi, C. Mahraoui, R. Benmessaoud et al., "Risk factors for a poor outcome among children admitted with clinically severe pneumonia to a university hospital in Rabat, Morocco," International Journal of Infectious Diseases, vol. 28, pp. 164170, 2014.

[19] WHO, Pocket Book of Hospital Cares for Children Guidelines for the Management of Common Illnesses with Limited Resources, WHO, Geneva, Switzerland, 2005.

[20] Food, Medicine and Health Care Administration and Control Authority (FMHACA), Standard Treatment Guideline for General Hospitals (2nd edition), Food, Medicine and Health Care Administration and Control Authority (FMHACA), Addis Ababa, Ethiopia, 2010.

[21] J. J. Babu, "Drug utilization evaluation of cephalosporins in general medicine units of rural tertiary care hospital," International Journal of Current Pharmaceutical Research, vol. 4, no. 2, pp. 88-91, 2012.

[22] D. F. Postma, C. H. van Werkhoven, L. J. R. van Elden et al., "Antibiotic treatment strategies for community-acquired pneumonia in adults," New England Journal of Medicine, vol. 372, no. 14, pp. 1312-1323, 2015.

[23] N. Eliakim-Raz, E. Robenshtok, D. Shefet et al., "Empiric antibiotic coverage of atypical pathogens for communityacquired pneumonia in hospitalized adults," Cochrane Database System Reviews, vol. 2012, no. 9, Article ID CD004418, 2012.

[24] L. Asadi, W. I. Sligl, D. T. Eurich et al., "Macrolide-based regimens and mortality in hospitalized patients with community-acquired pneumonia: a systematic review and metaanalysis," Clinical Infectious Diseases, vol. 55, no. 3, pp. 371-380, 2012.

[25] W. Nie, B. Li, and Q. Xiu, “ $\beta$-Lactam/macrolide dual therapy versus $\beta$-lactam monotherapy for the treatment of community-acquired pneumonia in adults: a systematic review and meta-analysis," Journal of Antimicrobial Chemotherapy, vol. 69, no. 6, pp. 1441-1446, 2014.

[26] M. Harris, J. Clark, N. Coote et al., "British Thoracic Society guidelines for the management of community acquired pneumonia in children: update 2011," Thorax, vol. 66, pp. ii1-ii23, 2011. 
[27] N. Le Saux and J. L. Robinson, "Uncomplicated pneumonia in healthy Canadian children and youth: practice points for management," Paediatrics \& Child Health, vol. 20, no. 8, pp. 441-445, 2015.

[28] K. Tiewsoh, R. Lodha, R. M. Pandey, S. Broor, M. Kalaivani, and S. K. Kabra, "Factors determining the outcome of children hospitalized with severe pneumonia," BMC Pediatrics, vol. 9, no. 1, p. 15, 2009. 